\title{
A Fuzzy Syntactic Method for On-Line Handwriting Recognition
}

\author{
Ashutosh Malaviya ${ }^{*}$ and Reinhard Klette ${ }^{* *}$ \\ * German National Research Center for Information Technology (GMD) \\ Schloß Birlinghoven, 53754 St. Augustin, Germany \\ ${ }^{* *}$ Computer Science Department, The Auckland University \\ Private Bag 92019, Auckland, New Zealand
}

\begin{abstract}
Fuzzy logic has proved to be a powerful tool to represent imprecise and irregular patterns. Thus the representation of handwriting parameters is accomplished with fuzzy linguistic modeling. The development of a multi-layered rule based handwriting recognition system is described. The selection of the representative features, which describe the shapes and location of segments, is the core of the proposed approach. Fuzzy linguistic terms provide these features the robustness against the handwriting variability. A new fuzzy language -FOHDEL for the syntactic description of on-line handwritten symbols is introduced.
\end{abstract}

\section{Introduction}

In recent years various syntactic handwriting recognition methods have been developed for both on-line and off-line applications [2][3][7][10]. Until now their success has been limited due to the large number of rules needed to cover the different handwriting styles. These difficulties partially originate from the crispness of the definition of the patterns, which in turn hinders the description of complexity and style variations of handwritten characters.

Considering the limitations and drawbacks of the existing systems we can define some major characteristics which a handwriting recognition system should possess: fast response, on-line adaptability, and flexibility. To achieve the fast responding and flexible recognition requirements, the designed method has to encompass a small but robust knowledge-base with which the incoming patterns are compared. Changes in style or orientation should be covered by the flexible prototypes which contain a widely valid description of character information. Linguistic description (rules) has this property, e.g. the character ' $b$ ' has a "very straight" "vertical line" in the "begin- 
ning" followed by an "almost" "circular curve" in the "end". On-line adaptability refers to the ability to incorporate new handwriting features of a writer during the recognition process itself. No matter how good the training phase is, there are always some unexpected features to which the given rules don't fit. To overcome this problem, there must be an automatic training method which just changes a part of, or extends, the knowledge base. There are several solutions which fit this requirement, like fuzzyneuro or genetic algorithm approaches [12].

First we demonstrate the significance of employing a fuzzy rule based approach for such problems with the example of human visual system. Following that we give some criteria for the multilevel fuzzy rule based pattern recognition method. The required fuzzy tools and an illustrative example are described in section 4 . We conclude with some general remarks related to the proposed method.

\section{Multi-level fuzzy rule based pattern recognition}

The uncertainties in the cognitive systems can have various reasons e.g. physical, random, statistical, chaotic and fuzzy. Depending upon the level of information cognition these uncertainties have corresponding effects. Therefore understanding or perception can be divided into various hierarchical levels. At the lower levels of understanding physical uncertainties are dominant because of the data acquisition inaccuracies. At the higher levels of understanding, other uncertainties come into picture which can have a fuzzy nature. The perception phenomena of physical objects can not always be explained by statistical measures and in some cases not at all. It is very difficult to classify the perception tasks rigidly into various uncertainty levels. A careful observation of biological recognition shows a close to perfect perception mechanism which functions amidst all sorts of uncertainties. This demonstrates that for a robust pattern recognition a methodology is required which deals with uncertainties in a more intelligent

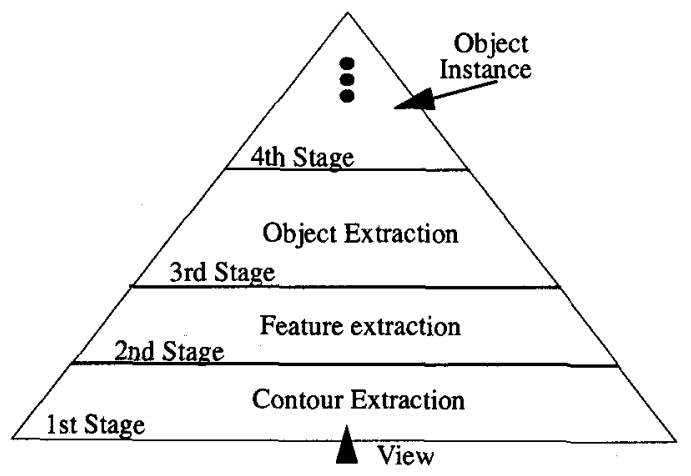

Figure 1 Multi-layered biological perception. 
manner especially at the higher levels of understanding. The anatomy of the primate form-processing pathway indicates that the visual system employs a multi-layered network [14]. The precise number of layers is debatable, but experiments have shown that normally there are seven or eight major cortical layers and at least four of them are employed for computation (see Fig. 1). The connections between the areas of primate visual form-processing also suggest that four computational stages are needed. The contour extraction and feature grouping is done at the first stage. The detection of relatively complex features at a number of retinal locations takes place during the second stage. The third stage involves the establishment of instances of approximate patterns or object features of a particular size and orientation, and subsequently in the final stage generalization across object instance in a view specific manner is accomplished. Moreover combination of the object parts and different views also occurs in this layer.

The first computational stage occurs early within the visual cortex and involves the first two cortical areas. The studies demonstrate that the boundaries between image regions and at the same time the logical groupings of local logical elements that belong together are computed. These processes differentiate the image attributes which belong to the same object. The other three cortical areas execute the second stage of computation. These areas also display sensitivity to an ever increasing complexity and variety of visual shape features (e.g. lines, corners, T-junctions, spotted triangle shape) [9]. The third stage processing utilizes combinations of these shape features. This establishes object-feature instances from vast amount of features. These object instances are then ordered according to the context of surroundings and other sensual information. It is very unlikely that all these stages can be achieved in a single step. Human brain builds up mental representation of real life situations layer by layer. The inner layer establishes the deepest aspect of the context. This also arises the question of modularity of knowledge representation. For instance if a person sees a new face, then most probably first the contour of the face image is extracted and then the facial features are detected and an object is identified. In the final stage actual face identification is accomplished. Naturally these theories are debatable and require further research. The scientific research of cognitive sciences until now has just produced some hints or clues regarding the perception and thinking process of humans.

Just in a similar manner as the face perception is described, the character recognition by people can also be explained. The necessary aspects of the characters are stored in the form of object instances. After the contours are extracted, the elementary features are generated. These again generate more complex features with the help of which character instances might be created. And as people see the known characters these object feature instances pop up and so the recognition is complete. This concept of biological systems was exploited in this work to divide the pattern recognition task into 
multiple layers and each of these layers creates a coded notion in form of rules for the following stage.

The human visual sense is selectively activated in response to curved lines and other geometrical characteristics [1]. Through this ability the human recognition system is the most accurate in grasping the typical features of the handwritten characters. These features themselves contain certain vagueness in terms of their definition. There have been some demonstrative experiments that indicate that the sharp contours are more discriminate than gradients, straight lines are more discriminate than the irregular squiggles. It is known that the brain does not store the entire picture or patterns of the world but only some aspects of it [14]. Recognition of the objects is then based on these aspects and not on their hard copies. Studies of neural networks and neural science in general try to decipher the code of internal workings of brain [14]. It has beendisclosed that some of the features are coded in the neurons, such as lines and edges of various orientations, and junctions and end points of contours. For human handwriting recognition the contextual clues play a very important role. It can be said that a human recognition system would produce much worse results in the absence of enough clues.

\section{Multi-level fuzzy rule based pattern recognition paradigm}

To compensate the uncertainties of pattern recognition systems a multi-level fuzzy rule based paradigm is presented. The inherent geometrical shape distortions of handwriting are primarily due to the variability in the handwriting styles and secondly due to the acquisition problems. Therefore the fuzzy set theory has been applied at various levels of uncertainties of handwriting recognition. The uncertainties are represented in terms of the membership to basic structural properties. Furthermore with fuzzy linguistic rules the complex handwriting patterns can be easily described in a broad linguistic domain, thus facilitating a flexible and widely valid recognition scheme.

The extraction of geometrical features can be described as the early processes in the visual cortex. The discovered codes of complex cells have shown the presence of bars and edges. These codes provide a compact representation of lines which is particularly appropriate to writing and printing. There are many handwriting recognition approaches which rely on geometrical and topological features. In many cases such feature strokes make individual letters [7]. The multi-layered rule base pattern recognition system (Fig. 3) in this work begins with the rule base segmentation of the preprocessed input handwriting profile in the first layer. In the following layer a set of basic features for each segment is computed. In the third layer features are further estimated employing the aggregation techniques [6]. The fuzzy aggregation methods support the integration of scattered and inconsistent information. A dedicated fuzzy language 


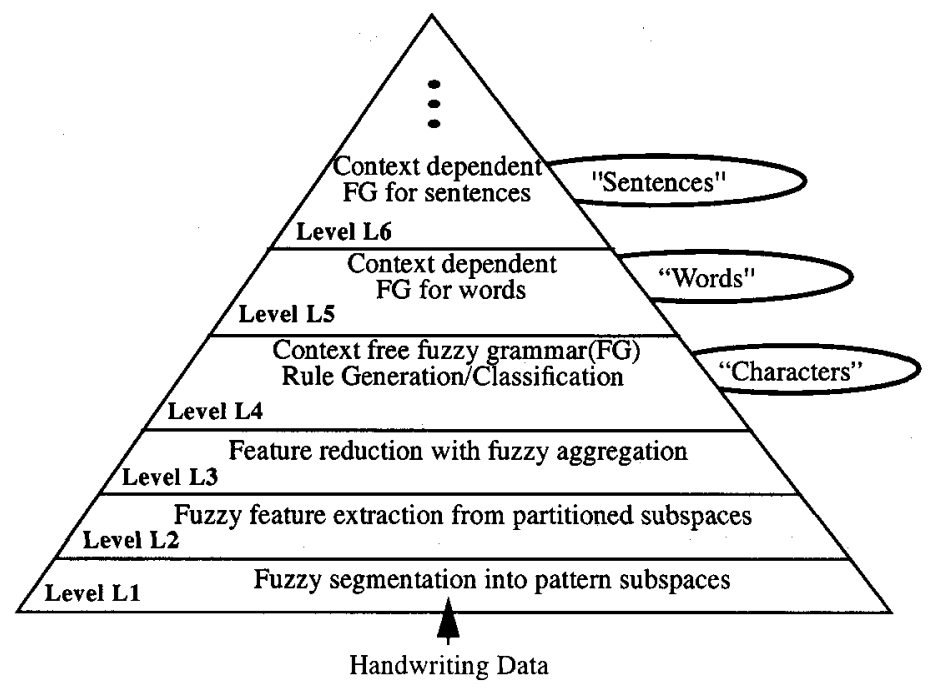

Figure 2 Multi-layered recognition scheme.

using attributed fuzzy grammars is developed to describe various symbols in the form of a rule-base. In the first phase of the fourth layer the generation of these rules is accomplished. And in the second phase of this layer the unknown characters are classified by parsing these rules. Assume the pattern space is partitioned into various fuzzy subspaces. These subspaces represent the domains of the local fuzzy features. The first layer of the rule-base is constructed by combining the extracted local features in terms of memberships. These memberships become then input for the next processing layer and the obtained conclusions are transmitted further as premisses for the next layer. These layers constitute a hierarchical network of the if-then type of rules (Fig. 3). At each level of this network an implication is made through the combination of various fuzzy objects, linguistic terms, modifiers and operators.

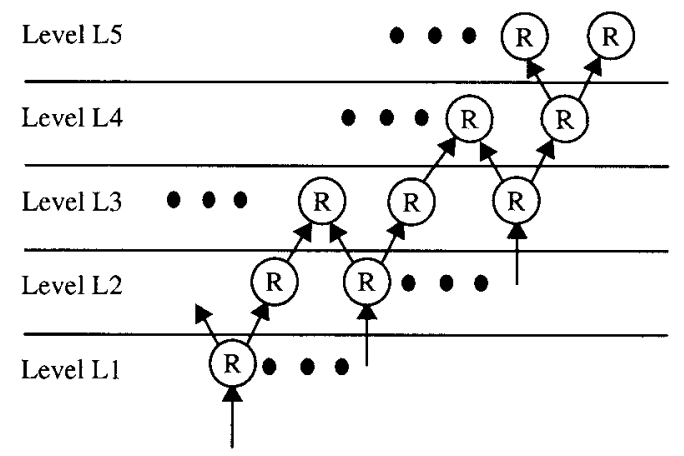

Figure 3 Multi-level rule base. 


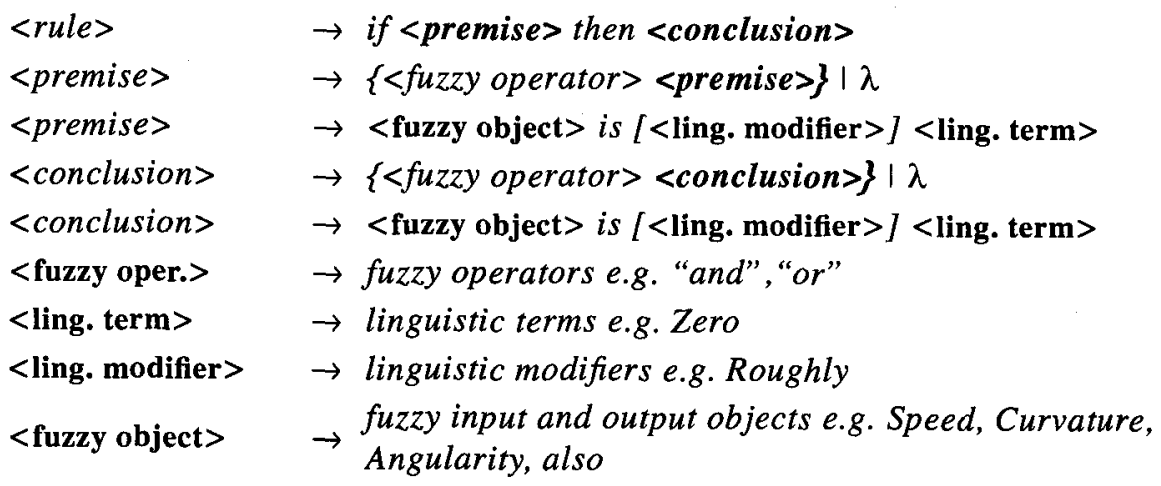

Now a formalism for multi-level linguistic rule base representation is shown (Fig. 4). The rules are derived from the described rule grammar.

$$
\begin{aligned}
& \text { Level L1: if } \overline{\text { Premise }}_{\mathrm{L} 1} \text { then } \overline{\text { Conclusion }}_{\mathrm{L} 1} \\
& \text { where } \overline{\text { Premise }}_{\mathrm{L} 1} \in \overline{\text { Conclusion }}_{\mathrm{L} 0} \\
& \text { Level L2: if } \overline{\text { Premise }}_{\mathrm{L} 2} \text { then } \overline{\text { Conclusion }}_{\mathrm{L} 2} \\
& \text { where } \overline{\text { Premise }}_{\mathrm{L} 2} \in \overline{\text { Conclusion }}_{\mathrm{L} 1} \\
& \text { Level Li: if } \overline{\text { Premise }}_{\mathrm{Li}} \text { then } \overline{\text { Conclusion }}_{\mathrm{Li}} \\
& \text { where } \overline{\text { Premise }}_{\mathrm{Li}} \in \overline{\text { Conclusion }}_{\mathrm{L}(\mathrm{i}-1)} \\
& \text { Level LN: if } \overline{\text { Premise }}_{\mathrm{LN}} \text { then } \overline{\text { Conclusion }}_{\mathrm{LN}} \\
& \text { where } \overline{\text { Premise }}_{\mathrm{LN}} \in \overline{\text { Conclusion }}_{\mathrm{L}(\mathrm{N}-1)}
\end{aligned}
$$

Where $\overline{\text { Premise }}_{\mathrm{Li}}$ is a set of premise at any level $\mathrm{j}<\mathrm{i}$ and $\overline{\text { Conclusion }}_{\mathrm{Li}}$ is a set of conclusions at $\mathrm{i}$-th level. $\mathrm{i} \in[0, \mathrm{~N}]$. And $\overline{\text { Premise }}_{\mathrm{L} 0}$ is empty. The processing of the levels $\mathrm{L} 1, \ldots, \mathrm{Li}, \ldots \mathrm{LN}$ is accomplished with fuzzy aggregation, rule inferencing and formal fuzzy grammars [8][11]. The choice of the applied technique depends on the semantic level and the possible syntactic relations. At the lower level, aggregation methods are more appropriate and at the higher level due to syntactic complexities fuzzy grammars are more suitable. Methods which describe characters in a linguistic form have been presented by various researchers over the last 30 years [2]. The linguistic techniques in pattern recognition are based on the structure of the underlying relationships between features in a two dimensional pattern. A complex pattern can be described in terms of basic primitives and sub-patterns. Now a formal multi-level linguistic rules structure is shown. The rules are derived from the described rule grammar. 


\begin{tabular}{|c|c|c|c|c|c|}
\hline $\begin{array}{l}\frac{\nabla}{\Delta} \\
\frac{0}{3} \\
\vdots\end{array}$ & R42: & $\begin{array}{l}\text { if } \\
\text { and } \\
\text { then } \\
\text { if } \\
\text { and } \\
\text { then }\end{array}$ & $\begin{array}{l}\text { vertical line left } \\
\text { O-like right } \\
\text { character ' } b \text { ' } \\
\text { vertical line left } \\
\text { D-like right } \\
\text { character ' } b \text { ' }\end{array}$ & $\begin{array}{l}\text { is } \\
\text { is } \\
\text { is } \\
\text { is } \\
\text { is } \\
\text { is }\end{array}$ & $\begin{array}{l}\text { very high } \\
\text { very high } \\
\text { very high } \\
\text { very high } \\
\text { high } \\
\text { very high }\end{array}$ \\
\hline$\frac{3}{3}$ & R31: & $\begin{array}{l}\text { if } \\
\text { and } \\
\text { then } \\
\text { if } \\
\text { and } \\
\text { then }\end{array}$ & $\begin{array}{l}\text { D-like } \\
\text { right-ness } \\
\text { D-like right } \\
\text { vertical line } \\
\text { left-ness } \\
\text { vertical line left }\end{array}$ & $\begin{array}{l}\text { is } \\
\text { is } \\
\text { is } \\
\text { is } \\
\text { is } \\
\text { is }\end{array}$ & $\begin{array}{l}\text { high } \\
\text { high } \\
\text { high } \\
\text { very high } \\
\text { high } \\
\text { very high }\end{array}$ \\
\hline 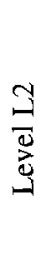 & R22: & $\begin{array}{l}\text { if } \\
\text { and } \\
\text { then } \\
\text { if } \\
\text { and } \\
\text { and } \\
\text { then }\end{array}$ & $\begin{array}{l}\text { left convexed-ness } \\
\text { arced-ness } \\
\text { D-like } \\
\text { straight-ness } \\
\text { arced-ness } \\
\text { vertical-ness } \\
\text { vertical line }\end{array}$ & $\begin{array}{l}\text { is } \\
\text { is } \\
\text { is } \\
\text { is } \\
\text { is } \\
\text { is } \\
\text { is }\end{array}$ & $\begin{array}{l}\text { high } \\
\text { very high } \\
\text { very high } \\
\text { very high } \\
\text { less than low } \\
\text { very high } \\
\text { high }\end{array}$ \\
\hline $\begin{array}{l}\bar{a} \\
\overline{8} \\
3 \\
\end{array}$ & R11: & $\begin{array}{l}\text { if } \\
\text { and } \\
\text { then } \\
\text { if } \\
\text { and }\end{array}$ & $\begin{array}{l}\text { relative curvature } \\
\text { relative velocity } \\
\text { new segment } \\
\text { relative angularity } \\
\text { relative velocity }\end{array}$ & $\begin{array}{l}\text { is } \\
\text { is } \\
\text { is } \\
\text { is } \\
\text { is }\end{array}$ & $\begin{array}{l}\text { more than high } \\
\text { less than low } \\
\text { very high } \\
\text { excellent } \\
\text { medium }\end{array}$ \\
\hline
\end{tabular}

Figure 4 Sample rule structure.

\section{Rule based handwriting recognition algorithm}

The multi-level rule-base paradigm proposed in the last section is implemented on the handwriting recognition system FOHRES ${ }^{1}$. The processing layers of the handwriting recognition are shown in Fig. 2 as a pyramid, which starts from the low level of data acquisition (L1) to the peak of the semantic identification (L5). The presented algorithm (Fig. 4) integrates the imprecision and the vagueness of the acquired handwritten symbols in various processing layers. The input to the bottom level $\mathrm{Ll}$ is the raw handwriting data, which is divided into various subspaces. To illustrate the proposed algorithm an example is given. The on-line handwriting is acquired from a pen-top.

1. Fuzzy On-line Handwriting REcognition System 
Algorithm 1 Fuzzy rule based handwriting recognition

Step 1: Divide the acquired on-line handwriting data space into smaller pattern domains like segments. The input parameters are the dynamics information including the relative curvature and the angularity (Level L1).

Step 2: Compute the geometrical features as fuzzy membership values for each domain (Level L2).

Step 3: Aggregate the features for all such domains to form global features (Level L3).

Step 4:

(a)Learning Phase: Form linguistic rules
with global linguistic features from Step 3
and integrate them in a fuzzy rule base
(Level L4).

(b)Classification Phase: Classify the unknown information by parsing the rule base created in Step 4(a), "Characters Level" (Level L4).

Step 5: Cross-check the "recognized" character in the given context. In case of error go to Step 4(b) for the next option. In case of failure go to Step 4(a) for on-line adaption. Else list recognized character (Level L5).

Figure 5 Fuzzy rule based handwriting recognition algorithm.

The on-line handwritten information is a list of coordinate pairs in a time sequence. These coordinates facilitate the evaluation of the pen motion dynamics like sudden changes of motion, jerks, high curvature etc. in a fuzzy linguistic manner. These parameters are termed as relative curvature, relative angularity and relative velocity. The partitioning of handwriting profile into fuzzy subspaces or segments is done with "if-then" rules from the evaluated dynamics information. During the processing level L2, fuzzy geometrical features for all segments are computed. The extracted features from these segments are combined to generate global features at the level L3. The combination of these fuzzy features is accomplished by employing fuzzy aggregation algorithms. In the following level L4, the relations between the extracted global features are represented in terms of linguistic knowledge representation with corresponding linguistic terms, modifiers and fuzzy operators. The classification is done with this knowledge representation. Following is an example demonstrating the proposed multilayered concept. A rule for the character ' $b$ ' is generated from the input co-ordinates (Fig. 4). Some ambiguous characters of ' $h$ ' and ' $b$ ' are then classified with this rule and another rule for ${ }^{h}$ ' is taken from the rule-base [12].

Example: Rule generation for the character ' $b$ ' from the on-line handwriting data and recognition from a confusing set of ' $b$ ' and ' $h$ ' characters. The input pattern in the form of coordinate pairs $\{x, y\}$ is given.

Pattern $=\{0,1\},\{0,0\},\{1,0\},\{1,2\},\{1,4\},\{1,6\},\{1,10\},\{1,13\},\{1,16\}$, $\{0,19\},\{1,20\},\{2,16\},\{3,15\},\{4,13\},\{10,11\},\{13,13\},\{13,15\}$, $\{12,18\},\{8,21\},\{6,22\},\{3,23\},\{2,23\},\{0,22\}$. 


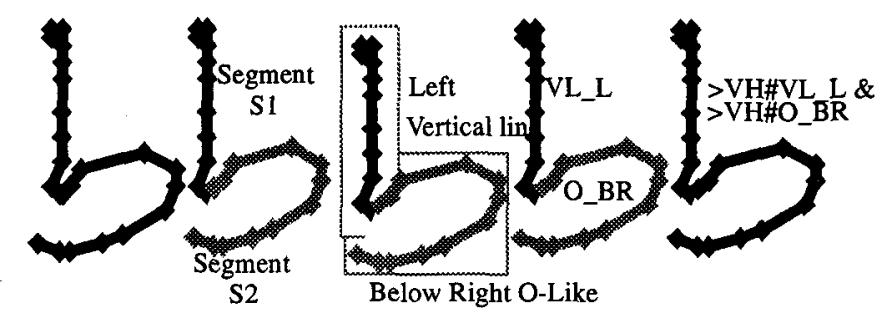
Level 0
Level 1
Level 2
Level 3
Level 4

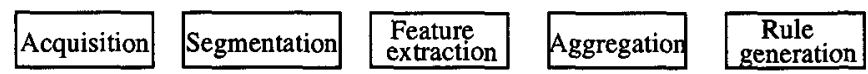

Figure 6 Processing levels of " $b$ ".

Step 1: The pattern is divided into segments ( $\mathrm{S} 1$ and $\mathrm{S} 2$ ) according to the fuzzy sharpness measures.

$\mathrm{S} 1=\{0,1\},\{0,0\},\{1,0\},\{1,2\},\{1,4\},\{1,6\},\{1,10\},\{1,13\},\{1,16\}$, $\{0,19\},\{1,20\}$

$S 2=\{2,16\},\{3,15\},\{4,13\},\{10,11\},\{13,13\},\{13,15\},\{12,18\},\{8,21\}$, $\{6,22\},\{3,23\},\{2,23\},\{0,22\}$

Step 2: For each of these segments the memberships to the following geometrical features are computed.

\begin{tabular}{l|l|l|l|l|l|l|l} 
Basic feature & Membership & $\mathrm{S} 1$ & $\mathrm{~S} 2$ & Basic & Membership & $\mathrm{S} 1$ & $\mathrm{~S} 2$ \\
\hline Straight-ness & $\mu_{\mathrm{SL}}$ & 0.86 & 0.07 & C-Like & $\mu_{\mathrm{CL}}$ & 0.27 & 0.05 \\
Arced-ness & $\mu_{\mathrm{Arc}}$ & 0.36 & 0.96 & D-Like & $\mu_{\mathrm{DL}}$ & 0.0 & 1.0 \\
Horizontal line & $\mu_{\mathrm{HSL}}$ & 0.0 & 0.0 & A-Like & $\mu_{\mathrm{AL}}$ & 0.27 & 0.55 \\
Verical line & $\mu_{\mathrm{VSL}}$ & 0.94 & 0.39 & U-Like & $\mu_{\mathrm{UL}}$ & 0.38 & 0.27 \\
Positive slant & $\mu_{\mathrm{PS}}$ & 0.0 & 0.40 & O-Like & $\mu_{\mathrm{OL}}$ & 0.01 & 0.80 \\
Negative slant & $\mu_{\mathrm{NS}}$ & 0.0 & 0.0 & Rel. & $\mu_{\mathrm{HP}}$ & 0.06 & 0.16 \\
Vertical curve & $\mu_{\mathrm{VC}}$ & 0.37 & 0.78 & Rel. & $\mu_{\mathrm{VP}}$ & 0.46 & 0.88 \\
Horizontal curve & $\mu_{\mathrm{HC}}$ & 0.03 & 0.20 & Relative & $\mu_{\mathrm{RL}}$ & 0.34 & 0.66
\end{tabular}

Table 1 Basic features membership values.

\begin{tabular}{|c|c|c|c|c|c|c|c|}
\hline Aggregated feature & Abbreviation & \multicolumn{2}{|c|}{ Membership } & Aggregated feature & Abbr. & \multicolumn{2}{|c|}{ Membership } \\
\hline Vertical line at left & $\mathrm{VL} \_\mathrm{L}$ & $\mu_{\mathrm{VL}_{2} \mathrm{~L}}$ & 0.83 & Relative number of segments & SEG & $\mu_{\mathrm{SEG}}$ & 0.40 \\
\hline O-like loop at bottom right & O_BR & $\mu_{\mathrm{O}} \mathrm{BR}$ & 0.95 & $X$ projection of End point & E_X & $\mu_{E} \mathrm{x}$ & 0.0 \\
\hline A-like curve at bottom right & A_BR & $\mu_{\mathrm{A} \_B R}$ & 0.30 & $X$ projection of Start point & $S_{-} X$ & $\mu_{\mathrm{S} \_\mathrm{x}}$ & 0.05 \\
\hline D-like curve at bottom right & D_BR & $\mu_{D_{-} B R}$ & 0.80 & $Y$ projection of End point & E_Y & $\mu_{E_{-} \mathrm{Y}}$ & 0.90 \\
\hline Relative number of pen-ups & PEN & $\mu_{\mathrm{PEN}}$ & 0.0 & $Y$ projection of Start point & S_Y & $\mu_{\mathrm{S}_{\mathrm{Y}} \mathrm{Y}}$ & 0.0 \\
\hline
\end{tabular}

Table 2 Aggregated features membership values. 
Step 3: With the help of a two phase aggregation scheme the membership values of various segments are combined to create feature primitives such as "vertical straight line"- VSL and "horizontal position"- HP and "relative length"- RL are combined into the global feature "Vertical line at left"VL_L. For the character ' $b$ ' the aggregated features are shown in Tab.2.

Step 4: (a) The extracted fuzzy features are finally written in the following FOHDEL ${ }^{2}$ language rule.

Rule b: Z\#PEN \& <L\#E_X \& M\#NSEG \& >VH\#VL_L\& ((H

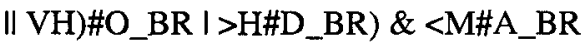

Interpretation: " $A$ symbol ' $b$ ' has no pen-ups (Z\#PEN) ${ }^{3}$ and ends at the beginning of the horizontal axis relative to the whole character ( $\angle \mathrm{LAE} \mathrm{X}$ ), has medium number of segments (M\#NSEG), has a very high or more ver. tical line at left (>VH\#VL_L), between high to very high $O$-like curve at bottom right ((HIIVH)\#O_BR), which can be a more than high D-like curve at bottom right (>H\#D_BR) and a medium or less likely an A-like curve at bottom right (<M\#A_BR)."

The transition of the classification of similar looking characters is now shown. This demonstrates how these FOHDEL features influence the classification. In this example a transition of the character ' $b$ ' to the character ' $h$ ' in a handwriting style is shown (Tab.4). The FOHDEL rule for ' $h$ ' is taken from the FOHDEL rule-base.

Rule h: Z\#PEN \& >L\#E_X \& M\#NSEG \& >VH\#VL_L \& (M\#O_BR \& >H\#A_BR) \& $<$ M\#D_BR

Interpretation: "A symbol ' $h$ ' has no pen-ups (Z\#PEN) and ends at the middle or end of the horizontal axis relative to the whole character ( $>\mathrm{L \# E} \mathrm{E}$ ), has medium number of segments (M\#NSEG), has a very high or more vertical line at left (>VH\#VL_L), a medium O-like curve at bottom right (M\#O_BR), also a high or more than high A-like curve at bottom right ( $>\mathrm{H \# A} \mathrm{A} B \mathrm{BR})$ and medium or less likely a D-like curve at bottom right (M\#D_BR)."

\begin{tabular}{c|l|c|l} 
Symbol & \multicolumn{1}{|c|}{ FOHDEL Operators } & Symbol & \multicolumn{1}{|c}{ FOHDEL Operators } \\
\hline$\#$ & Separator between linguistic term and feature & $\|$ & BETWEEN modifier \\
$\&$ & AND operation i.e. MIN operation & $>$ & MORE THAN modifier \\
1 & OR operation i.e. MAX operation & $<$ & LESS THAN modifier
\end{tabular}

Table 3 FOHDEL operators.

2. Fuzzy On-Line Handwriting DEscription Language

3. Z,VVL, VL,L,M,H,VH,VVH,E correspond to linguistic terms Zero, Very Very Very Low, Very

Low, Medium, High, Very High, Very Very High and Excellent. 


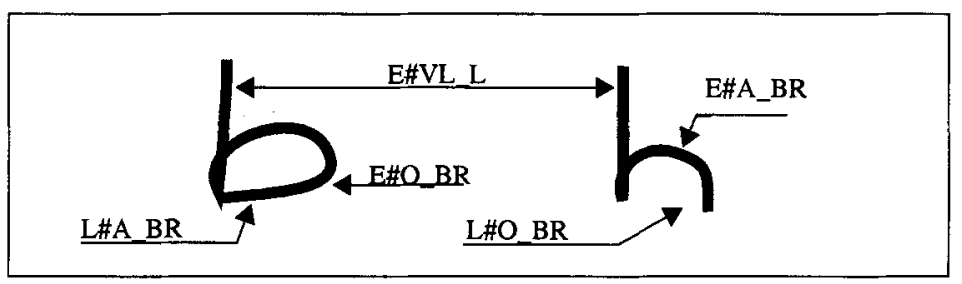

Figure 7 Discrimination criteria.

Step 4: (b) The classification results of five characters based on the rules extracted in step 4(a) with a fuzzy inference process is shown in Tab.4.

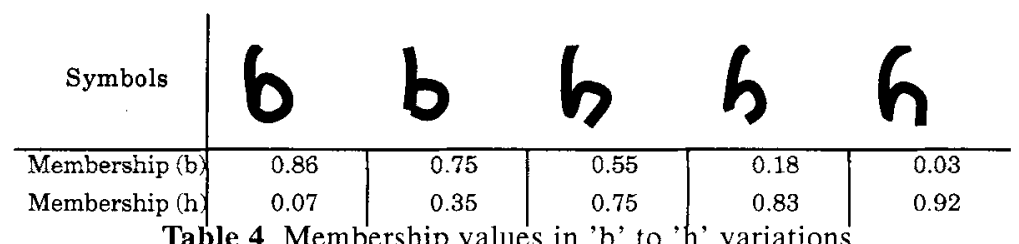

Table 4 Membership values in ' $b$ ' to ' $h$ ' variations.

Step 5: The possibility of the unknown character to be symbol ' $b$ ' decreases from left to right while the possibility of the input character of being ' $h$ ' increases correspondingly. The discrimination between ' $b$ ' and ' $h$ ' is done on the basis of "A-Like" and "O-Like" features (Fig. 7).

\section{Summary}

A multi-level recognition methodology is proposed which is analogous to neuro-biological findings regarding perception. The psychological studies of human handwritings have given interesting clues regarding feature extraction and syntactical relations. Some of these results are incorporated to support the recognition mechanism. The aggregation scheme is analogous to the stimulus generation as the means of feature reduction and selection of only representative features. The proposed multi-layered recognition model has helped to tune the recognition system in a structured way. The parameters could be studied qualitatively which ultimately influence the handwriting recognition performance. The classification stage offered multiple outputs as a list of priorities (membership function). The experimental results [13] have shown that FOHRES can reduce many of the existing ambiguities in handwritten symbols and has enough flexibility to adapt to the variations of handwriting styles.

\section{References}

[1] J. F. Baldwin and B. W. Pilsworth, "Axiomatic Approach to Implication for Approximate Reasoning with Fuzzy Logic,'” Fuzzy Sets and Systems, Vol. 3, pp. 193-219, 1980. 
[2] K.S. Fu, Syntactic Pattern Recognition and applications, NJ, Prentice-Hall, 1982.

[3] M.T. Gary et al,"A Fuzzy-Attributed Graph Approach to Handwritten Character Recognition," FUZZ-IEEE-93, pp. 570-575, 1993.

[4] I. Guyon et al., "UNIPEN project of on-line data exchange and recognition benchmarks," 13th IEEE-ICPR, pp. 29-33, Israel, 1994.

[5] J.A. Jorge,"Fuzzy Relational Grammars for Interactive Gesture Recognition,"2nd International Conf. on Fuzzy Set Theory and Technology, Durham, NC, Oct.13-16, 1993.

[6] J. M. Keller et al.,"Evidence Aggregation networks for fuzzy logic inference," IEEE T. on Neural Networks, vol.3, No.5,pp.761-769,Sept. 1992.

[7] M.-S. Lan et al,"Character Recognition using Fuzzy Rules Extracted from Data," FUZZ-IEEE-94, pp.415-420, Orlando, June,1994.

[8] E.T. Lee and L.A. Zadeh, "Note on Fuzzy Languages," Information Sciences-1, pp. 421-434, 1969.

[9] R.Klette and P. Zamperoni, Handbook of Image Processing Operators, Wiley, Chichester, 1996.

[10] C. Leja, Entwicklung und Verifikation von intelligenten Lernverfahren für regelbasierte Systeme sowie einer geeigneten graphischen Benutzeroberfläsche, Diplomarbeit, Uni. Siegen, October, 1995.

[11] A. Malaviya et al,"FOHDEL - a fuzzy handwriting description language," FUZZ-IEEE, June 1994.

[12] A. Malaviya et al,"Automatic generation of fuzzy rule base for online handwriting recognition," EUFIT-94, Aachen, 1994.

[13] A. Malaviya and L. Peters,"Extracting meaningful handwriting features with fuzzy aggregation method,"3rd Intnl' Conf. on Document Analysis and Recognition, Montreal Canada, 1995.

[14] M.W. Oram and D.I. Perrett,"Modeling visual recognition from neurobiological constraints," Neural Networks, vol.7, No.6,7, pp.945-972, 1994.

[15] A.C. Shaw, "A Formal Picture Description Scheme as a Basis for Picture Processing Systems," Information and Control-14, pp. 9-52, 1969.

[16] A. Sloman,"On designing a visual system (Towards a Gibbsonian computational model of vision),"J.Exp.Theor.A.I., No.1 pp.289-337,1989.

[17] K.C. Yau and K.S. Fu,"A Syntactic Approach to Shape Recognition Using Attributed Grammars," IEEE-SMC-9, No. 6, pp. 334-345, 1979. 